\title{
Agôn
}

Revue des arts de la scène

Critiques | Saison 2016-2017

\section{Chunky Charcoal, de Sébastien Barrier, Benoît Bonnemaison-Fitte et Nicolas Lafourest}

Pertes et mots

\section{Caroline Châtelet}

\section{(2) OpenEdition}

Journals

Édition électronique

URL : http://journals.openedition.org/agon/4013

DOI : 10.4000/agon.4013

ISSN : 1961-8581

Éditeur

Association Agôn

\section{Référence électronique}

Caroline Châtelet, "Chunky Charcoal, de Sébastien Barrier, Benoît Bonnemaison-Fitte et Nicolas

Lafourest », Agôn [En ligne], Critiques, mis en ligne le 07 février 2017, consulté le 23 septembre 2020.

URL : http://journals.openedition.org/agon/4013; DOI : https://doi.org/10.4000/agon.4013

Ce document a été généré automatiquement le 23 septembre 2020

Association Agôn et les auteurs des articles 


\title{
Chunky Charcoal, de Sébastien Barrier, Benoît Bonnemaison-Fitte et Nicolas Lafourest
}

Pertes et mots

\author{
Caroline Châtelet
}

\section{RÉFÉRENCE}

Chunky Charcoal, de Sébastien Barrier, Benoît Bonnemaison-Fitte et Nicolas Lafourest présenté au théâtre de La Colline du 5 au 28 janvier 2017

1 Il y a des projets qui, tout en travaillant un dispositif modeste, parviennent à déployer une forme aussi labyrinthique qu'inventive. Chunky Charcoal, nouveau projet de Sébastien Barrier, est de ceux-là, à tel point que la matière de la création excède autant sa structure qu'elle l'alimente. Figure un brin iconoclaste, autant comédien-conteur que conteur-comédien - le régime de parole n'étant pas tout à fait le même dans l'une ou l'autre des situations - Sébastien Barrier est connu dans le champ des arts de la rue pour son personnage de Renan Tablantec. Un pêcheur breton et raconteur invétéré d'histoires, qui a au fil des ans pris de plus en plus de place dans la vie de Sébastien Barrier. Si cette aliénation du comédien à son personnage, voire, leur fusion, ne sont peut-être pas innocentes dans le choix de Barrier de "tuer» son alter-ego, cette contamination de l'un par l'autre en dit long sur la qualité particulière de présence de l'acteur. Barrier est un homme qui parle, et qu'il soit pêcheur breton dans la rue ou Sébastien Barrier sur la scène du théâtre national de La Colline, qu'il réalise une dégustation de vin (comme dans Savoir enfin qui nous buvons, spectacle marathonoenologique) ou narre les bribes de mille et une vies comme dans Chunky Charcoal, son outil est la langue. Une langue ininterrompue, proliférante, drôle, touchante, etc. Pour accompagner celle-ci, un dispositif et des acolytes sont réunis: tandis qu'un vaste panneau de papier blanc va accueillir au fil du spectacle les dessins de Benoit 
Bonnemaison-Fitte, des instruments de musique à l'avant et côté cour de la scène - où se trouve également le musicien Nicolas Lafourest - seront utilisés par Lafourest et Barrier.

De ce spectacle, il était dit sur le site internet de la Colline qu'il s'agissait d'une proposition «poético-rupestre ». Un qualificatif étrange voire, inquiétant, le "poétique» étant parfois convoqué pour qualifier une forme ou un propos difficilement identifiables, quand il ne s'agit pas de masquer leur vacuité (dans ce second cas, c'est souvent l'échec, ça ripoline pas terrible en fait la poésie). Dans ce travail, cela ne ripoline rien du tout, et s'avère au final d'une pertinence indubitable. Du village d'origine de Benoit Bonnemaison-Fitte - Aurignac, berceau de l'aurignacien, culture du paléolithique supérieur - à sa rencontre avec son chat - présent dans le spectacle -, des personnes croisées en tournée à celles, mortes désormais, qu'ils ne reverront plus, de toutes ces histoires enchâssées une poésie se dégage. Une poésie du quotidien, de l'intime, de ces petits récits et anecdotes qui font des vies, les peuplent et les dépeuplent, les enjolivent et parfois les abîment. Au gré des récits glanés, des récurrences et des liens se font jour : il y a la question de la perte; de la disparition (des sentiments, des personnes, des époques); de ce qui échappe, comme le dit si bien cette phrase répétée plusieurs fois "Tout ce qui échappe à notre regard est-il perdu pour autant?»; mais également celle de la relation. Avec son verbe luxuriant, ses histoires qui resurgissent et s'enchevêtrent, Sébastien Barrier met en relation des mondes, des récits, des mots, accompagné et soutenu par le travail graphique de Benoit Bonnemaison-Fitte. Écrivant à l'aide de craies noires (de la marque chunky charcoal) avec des tailles, typographies et lettres différentes certains termes prononcés, le dessinateur les relève, méticuleux, et les relie lui aussi. D'autres résonances apparaissent entre les histoires, parfois souterraines, quelquefois évidentes, avec des creux, des absences, de ces petits mystères peuplant des existences entières. Génial par son inventivité et sa vivacité, ce work in progress - dont on comprend qu'il ne cesse de s'enrichir au fil des tournées - construit sa galaxie et traverse les territoires comme les périodes historiques. Jusqu'à ce fameux art rupestre, évoqué en préambule du spectacle et qui alimente et relie l'ensemble. Entremêlant conte, musique, dessin et écriture, Chunky Charcoal devient alors la mise en jeu de possibles hypothèses quant à l'interrogation initiale de Sébastien Barrier sur la fonction de l'art rupestre. À quoi servait-il? Nul ne le sait. En nous demeurant éternellement inaccessible dans sa fonction, cette production renvoie aussi aux parts manquantes, inachevées des vies esquissées par le comédien, comme, plus largement au rôle de l'art dans notre société. 
Chunky Charcoal, de Sébastien Barrier, Benoît Bonnemaison-Fitte et Nicolas Lafourest

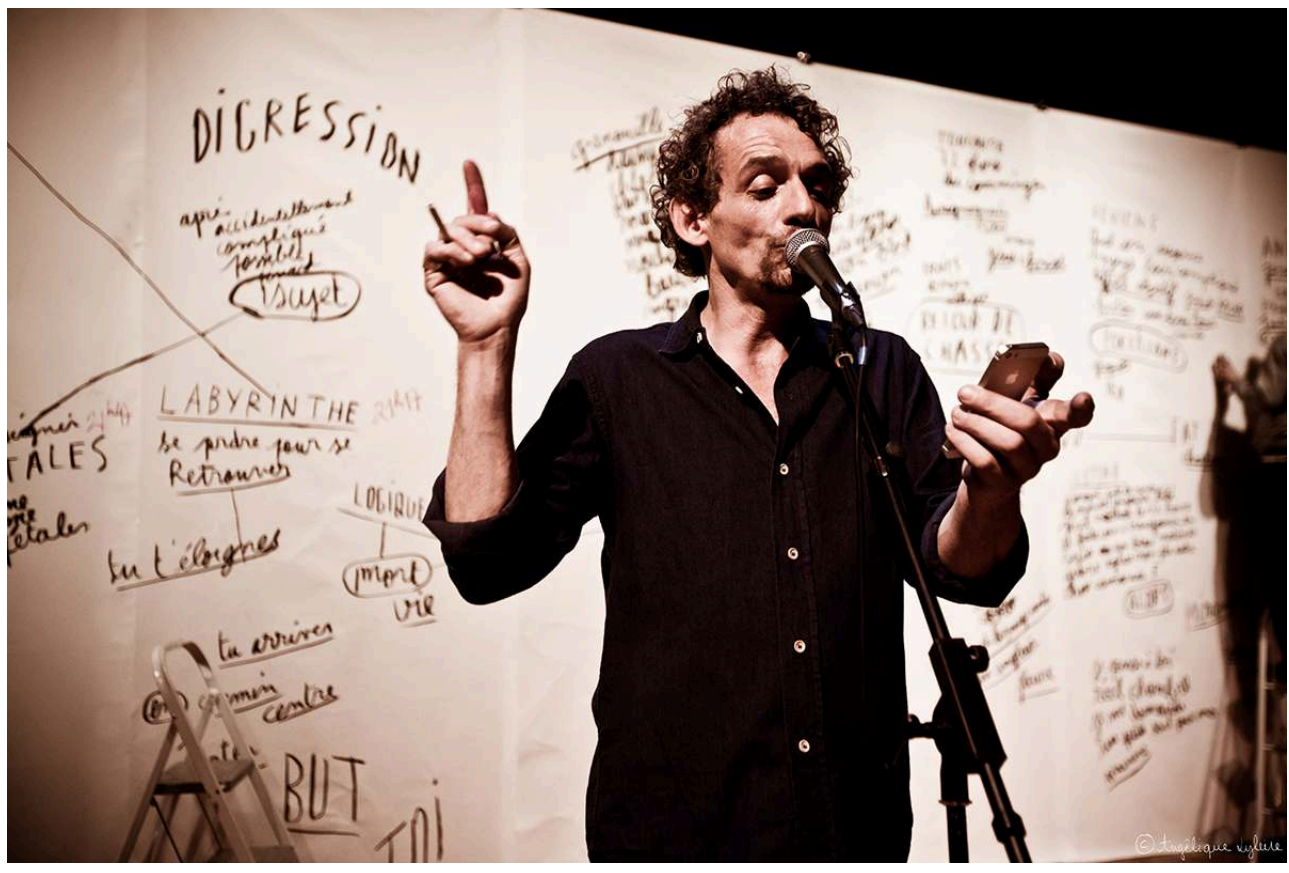

Angélique Lyleire 